\title{
The relation between age and outcomes of thoracic sympathectomy for hyperhidrosis: The older the better
}

\author{
Dafne Braga Diamante Leiderman, MD, ${ }^{\mathrm{a}}$ Jose Ribas Milanez de Campos, MD, PhD, ${ }^{\mathrm{b}, \mathrm{c}}$ \\ Paulo Kauffman, MD, PhD, ${ }^{\mathrm{a}, \mathrm{d}}$ Miguel Lia Tedde, MD, PhD, ${ }^{\mathrm{c}}$ Guilherme Yazbek, MD, PhD, \\ Marcelo Passos Teivelis, $\mathrm{MD}, \mathrm{PhD},{ }^{\mathrm{a}}$ and Nelson Wolosker, $\mathrm{MD}, \mathrm{PhD}^{\mathrm{a}, \mathrm{d}}$
}

\begin{abstract}
Objective: Several factors may potentially influence the efficacy and patient satisfaction after bilateral thoracic sympathectomy as the treatment for hyperhidrosis, but few studies have specifically analyzed the impact of age on the efficacy of this treatment, the occurrence of compensatory hyperhidrosis $(\mathrm{CH})$, and variations in the quality of life.

Methods: We retrospectively analyzed the effect of age, body mass index, surgical techniques, quality of life before surgery, betterment in the quality of life after surgery, clinical improvement in sweating at the main site, and the occurrence and intensity of $\mathrm{CH}$ in patients with hyperhidrosis $(\mathrm{n}=1633)$ who underwent bilateral sympathectomy.
\end{abstract}

Results: Quality of life improved in more than $90 \%$ of patients, and severe $\mathrm{CH}$ occurred in 5.4\%. Age did not affect these outcomes. The older, the greater reduction in sweating, and $\mathrm{CH}$ was linked to other variables (body mass index, craniofacial hyperhidrosis, and level of resection).

Conclusions: We observed that patients with old age reported an improvement in sweating in the main site of hyperhidrosis. Sympathectomy outcomes in older patients are similar to those observed in younger patients in terms of quality of life improvement and occurrence of $\mathrm{CH}$. (J Thorac Cardiovasc Surg 2018;156:1748-56)

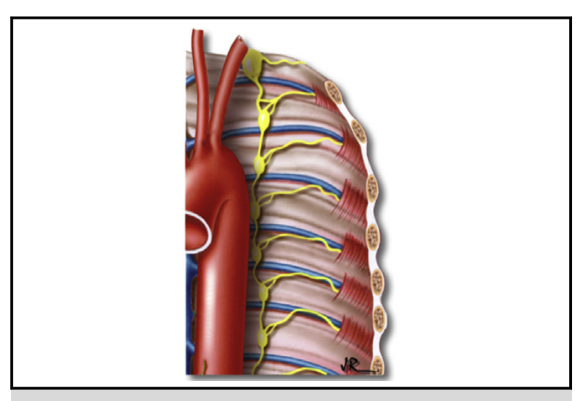

Sympathetic chain (in yellow)

\section{Central Message}

Sympathectomy for hyperhidrosis at an old age shows improvement in excessive sweating without increased compensatory hyperhidrosis

\section{Perspective}

We investigated the association between age and sympathectomy response in a large group of patients with hyperhidrosis $(\mathrm{n}=1633)$ and observed that as age increases, patients experience an improvement in excessive sweating with no increase in the frequency of compensatory hyperhidrosis. Our results support that old age should not be considered a negative characteristic for sympathectomy candidates.

See Editorial Commentaries pages 1757 and 1759.
Hyperhidrosis has a major impact on quality of life, affecting patients' personal and professional relationships. ${ }^{1,2}$ In most cases, symptoms of hyperhidrosis first

\footnotetext{
From the ${ }^{\mathrm{a}}$ Department of Vascular and Endovascular Surgery and ${ }^{\mathrm{b}}$ Division of Thoracic Surgery, Hospital Israelita Albert Einstein, São Paulo, São Paulo, Brazil; ${ }^{\mathrm{c}}$ Thoracic Surgery Division and ${ }^{\mathrm{d}}$ Vascular and Endovascular Division, Department of Surgery, University of São Paulo School of Medicine, São Paulo, São Paulo, Brazil; and ${ }^{\mathrm{e}}$ Department of Vascular and Endovascular Surgery, A.C. Camargo Cancer Center, Fundação Antônio Prudente, São Paulo, São Paulo, Brazil.

Received for publication Sept 11, 2017; revisions received May 7, 2018; accepted for publication May 9, 2018; available ahead of print July 24, 2018.

Address for reprints: Dafne Braga Diamante Leiderman, MD, Av Albert Einstein, 627, Rm 423/ A1 Building, São Paulo, São Paulo 05652-000, Brazil (E-mail: dah.diamante@gmail.com).

$0022-5223 / \$ 36.00$

Copyright (c) 2018 by The American Association for Thoracic Surgery

https://doi.org/10.1016/j.jtcvs.2018.05.084
}

appear in childhood and adolescence and persist throughout adulthood. As hyperhidrosis has a significant negative impact on the quality of life, patients tend to seek intervention earlier in life and to undergo surgery in their teenage years or early adulthood, ${ }^{3,4}$ but mostly during their economically active ages. ${ }^{5,6}$

Video-assisted thoracic surgery (VATS) sympathectomy is considered the gold standard for definitive hyperhidrosis

- Scanning this $\mathrm{QR}$ code will take you to a supplemental video for the article. 

Abbreviations and Acronyms
BMI = body mass index
$\mathrm{CH}=$ compensatory hyperhidrosis
$\mathrm{CI}=$ confidence interval
$\mathrm{OR}=$ odds ratio
VATS $=$ video-assisted thoracic surgery

treatment in patients whose clinical improvement with medication is unsatisfactory. ${ }^{2}$ Several factors may influence the efficacy and patient satisfaction after bilateral VATS sympathectomy as a treatment for hyperhidrosis, ${ }^{1}$ including body mass index (BMI), ${ }^{2}$ resection level,,${ }^{7,8}$ and number of resected ganglion. ${ }^{9}$ To the best of our knowledge, there are no studies in the literature that assess whether an older age is a negative factor for quality of life improvement after surgery or the development of compensatory hyperhidrosis ( $\mathrm{CH}$; also known as compensatory sweating). Based on our clinical experience, we speculated that older patients could experience worse sympathectomy results than younger patients. The aim of this study was to investigate the association between age and treatment response to VATS sympathectomy in a large group of patients with hyperhidrosis by analyzing several parameters, including the reduction in sweating, compensatory sweating, and quality of life in patients.

\section{METHODS}

We retrospectively analyzed 1633 patients who underwent bilateral VATS sympathectomy as a treatment for hyperhidrosis from January 2000 to April 2014 in a specialized ambulatory care setting. This study was approved by the research ethics committee of the institution.

In 2008, our team started a research project using oxybutynin as a treatment for hyperhidrosis. ${ }^{10}$ Based on the positive results obtained in that study, our protocol was modified in 2011, and all patients underwent clinical treatment with oxybutynin. Of those, only patients who had poor improvement in sweating (or developed bothersome side effects) underwent surgery. Therefore, patients included in this study were subjected to sympathectomy as first-line treatment (before 2008) or after failed clinical treatment with oxybutynin (after 2008). After surgical treatment, no patients were administered oxybutynin within the first 30 days (a small number of patients used oxybutynin afterwards for treatment of $\mathrm{CH}){ }^{11}$

All patients underwent a procedure that involved making 2 mini-incisions of $1 \mathrm{~cm}$ in length over each hemithorax: one on the anterior axillary line in the third or fourth intercostal space and the other on the midaxillary line in the second or third intercostal space. Whenever needed, a third incision was made to facilitate the approach to the sympathetic chain. All procedures were performed with the patient under general anesthesia with simple intubation in cases of resection at the G2 and/or G3 ganglion levels and selective intubation of the lungs at more caudal levels, or when additional difficulties in the dissection of the sympathetic chain were anticipated. Patients were in a semiseated position with 2 small subscapular cushions placed to position the upper limbs in abduction at $90^{\circ}$, one cushion below the knees, and an abdominal belt to avoid limiting the lateral motion of the surgery table. ${ }^{12}$

The approach level was defined according to the patient's most prominently localized complaint area, which was always between the G2 and G4 ganglia. The targeted level of resection changed during the 15-year period. We progressively performed fewer sympathectomies at higher levels aiming to reduce $\mathrm{CH}$. In the last 5 years, we have preferentially performed ablation of $\mathrm{G} 2$ for craniofacial hyperhidrosis only, G3 or G4 for palmar, G4 for axillary, and G4 for the combination of palmar and axillary only. If the patient had craniofacial-associated palmar hyperhidrosis, ablation of the G2 level was preferred. If the patient had axillary hyperhidrosis in addition to facial hyperhidrosis, resection of $\mathrm{G} 2, \mathrm{G} 3$, and G4 ganglia was performed but at a high risk of severe $\mathrm{CH} .{ }^{12}$

Sympathectomy was mainly performed by thermoablation, using a harmonic or electric scalpel. Resection was performed only at the beginning of our study period but was replaced by thermoablation to reduce the surgical risk of bleeding while achieving similar results. All our patients were admitted for surgery and scheduled for discharge the next morning (Video 1). ${ }^{12}$

All patients treated at our service have been routinely evaluated since 2000 with regular completion of a specific medical record, which included demographic data, descriptions of all sites of hyperhidrosis, 2 specific quality of life questionnaires, ${ }^{13,14}$ the Sweating Evolution Questionnaire, ${ }^{15}$ and the occurrence of $\mathrm{CH}$.

In this study, we analyzed age as a continuous variable to assess whether it influenced surgical outcomes. Other variables included sex, BMI (also a continuous variable), main hyperhidrosis site, and sympathectomy techniques.

The primary endpoints in the study were as follows:

1. Patient's quality of life at the first visit before surgery; and

2. Patient's perception of the degree of betterment in the quality of life after surgery, clinical improvement in sweating, and the occurrence of $\mathrm{CH}$ after the first postoperative month.

Quality of life was measured via a protocol described by Amir and colleagues $^{16}$ and translated into Portuguese by de Campos and colleagues. $^{13,14}$ This protocol comprises 20 questions divided into 4 domains (functional-social, personal, emotional, and special conditions). Only 1 answer is allowed for each question. These quality of life assessments were completed by the patient before surgery, without the involvement of a physician. ${ }^{13,14,16}$

Before surgery, patients described their feelings regarding the 4 domains with 5 levels of response ("excellent," "very good," "good," "bad," and "very bad"). Patients were then classified into 5 different quality of life levels that were calculated as the sum total score of the protocol, ranging from 20 to 100 . A sum greater than 83 was classified as "very poor quality of life," 68 to 83 as "bad," 52 to 67 as "good," 36 to 51 as "very good," and 20 to 35 as "excellent"13,14 (Online Data Supplement).

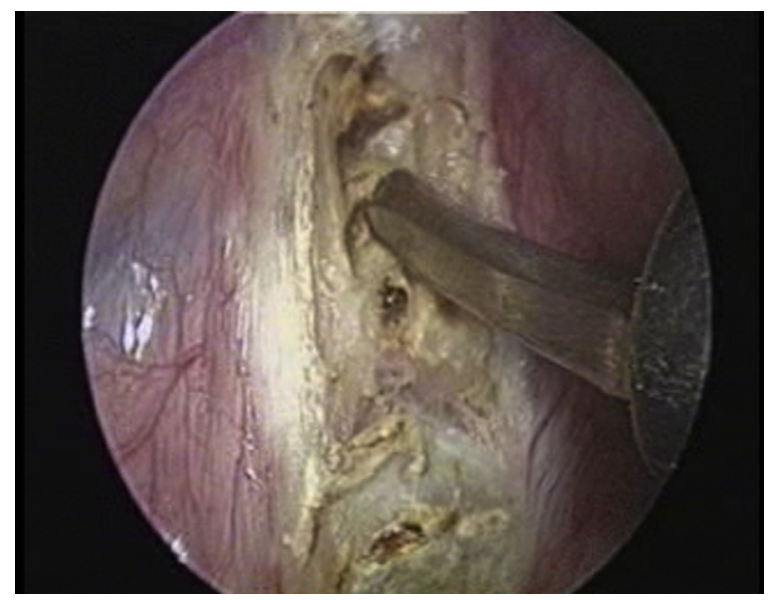

VIDEO 1. Video thoracoscopic sympathectomy. Video available at: https://www.jtcvs.org/article/S0022-5223(18)31519-8/fulltext. 
After surgery, patients completed the second part of the questionnaire, which evaluated the subjective betterment in the quality of life considering the same 4 domains assessed in the first part of the questionnaire. ${ }^{13,14,16}$ Patients also answered with 5 levels of response ("much better," "better," "equal," "worse," "much worse"). The levels of betterment were then calculated as the sum total score of the protocol: a sum greater than 83 was classified as a "much worse quality of life after surgery," 68 to 83 as a "little worse," 52 to 67 as "equal," 36 to 51 as a "little better," and 20 to 35 as "much better" 13,14 (Online Data Supplement).

The postoperative and preoperative protocols were not the same-these protocols are similar but the question and the meaning of the answers are different, that is why it was not possible to calculate a "delta" of the 2 questionnaires scores.

The clinical improvement in sweating after treatment was assessed with the Sweating Evolution Questionnaire, ${ }^{15}$ an analogic and quantification scale ranging from 0 to 10 , in which 0 represents no improvement in sweating and 10 represents the absence of sweat (anhidrosis) for each site of hyperhidrosis based on patient self-assessments. Clinical improvement was graded as follows: null (0-4), moderate (5-7), and great (8-10), based on the number regarding the patient's main complaint.

To analyze the incidence of $\mathrm{CH}$, we used the patient's report confirmed by physical examination. $\mathrm{CH}$ was graded as severe and nonsevere according to the following: severe-visible $\mathrm{CH}$, embarrassing for the patient, and the patient needed to change clothes more than once throughout the day ${ }^{17}$; nonsevere-visible $\mathrm{CH}$ and embarrassing for the patient but not severe enough to require a change of clothes (moderate $\mathrm{CH}$ ), or visible and embarrassing only sometimes (hot weather and during exercise-mild $\mathrm{CH}$ ). ${ }^{17}$

Normality was assessed with the Kolmogorov-Smirnov test and visual inspection. Variables (age and BMI) were not normally distributed and are presented as medians and quartiles. Qualitative characteristics were described using absolute and relative frequencies. Logistic regression bivariable was used to verify the association between the quality of life, $\mathrm{CH}$, severe $\mathrm{CH}$, and clinical improvement and the qualitative and quantitative variables. Only the significant variables $(P<.05)$ of the bivariable analysis were inserted in the multivariable analysis models and only variables selected by the stepwise backward criterion were kept in the final models presented on the tables. Odds ratios (ORs) were calculated with confidence intervals (CIs) for each variable according to the outcomes using bivariable and multivariable logistic regression. Three patients (2 G4/G5 and 1 G2/G3/G4) were excluded from the statistical analysis in all models, because the statistic models were overfit and those categories had low number of patients. All statistical analyses were performed with IBM-SPSS for Windows 20.0 (IBM Corp, Armonk, NY); all tests were performed with a significance level of $5 \%$.

\section{RESULTS}

We analyzed data from 1633 patients who underwent VATS sympathectomy as a treatment for hyperhidrosis. The median age of the patients was 25 years old (interquartile range 21-30 years; range 18-70 years old), and the median BMI was $21.67 \mathrm{~kg} / \mathrm{m}^{2}$ (interquartile range $20-31 \mathrm{~kg} /$ $\mathrm{m}^{2}$; range $17.9-32.27 \mathrm{~kg} / \mathrm{m}^{2}$ ).

Table 1 shows the association of sex, main site of hyperhidrosis, level of resection in VATS sympathectomy, and age of symptom onset with patient's age. Patients with craniofacial hyperhidrosis and onset of symptoms in adulthood were older than others with hyperhidrosis as the main site and onset symptoms in childhood or adolescence, respectively.

Baseline and outcomes data are presented in Table 2. All patients had a "poor" $(\mathrm{n}=320 ; 19.6 \%)$ or "very poor" quality of life ( $\mathrm{n}=1313 ; 80.4 \%)$ before surgery.
TABLE 1. Age distribution among clinical and operative variables

\begin{tabular}{|c|c|c|c|}
\hline Variables & $\mathbf{N}(\%)$ & $\frac{\text { Age, } y}{\text { Median }(p 25 ; \text { p75) }}$ & $P$ value \\
\hline Sex & & & .349 \\
\hline Female & $1095(67.1)$ & $25(22 ; 30)$ & \\
\hline Male & $538(32.9)$ & $25(21 ; 30)$ & \\
\hline Main hyperhidrosis site & & & $<.001 *$ \\
\hline Palmar $\dagger, \ddagger$ & $1013(62)$ & $24(21 ; 29.5)$ & \\
\hline Axillary $\ddagger$ & $569(34.8)$ & $26(22 ; 32)$ & \\
\hline Craniofacial $\dagger, \S, \|$ & $31(1.9)$ & $31(24 ; 42)$ & \\
\hline Plantar & $20(1.2)$ & $25(22 ; 29)$ & \\
\hline Technique & & & $.103^{*}$ \\
\hline $\mathrm{G} 2$ & $69(4.2)$ & $26(22 ; 33)$ & \\
\hline G3 & $532(32.6)$ & $25(21 ; 29)$ & \\
\hline G4 & $576(35.3)$ & $26(22 ; 31)$ & \\
\hline G2/G3 & $258(15.8)$ & $25(21 ; 31)$ & \\
\hline G3/G4 & $184(11.3)$ & $24.5(21 ; 32)$ & \\
\hline G4/G5 & $2(0.1)$ & & \\
\hline $\mathrm{G} 2 / \mathrm{G} 3 / \mathrm{G} 4$ & $1(0.1)$ & & \\
\hline No information & $11(0.7)$ & & \\
\hline Age of symptom onset & & & $<.001 *$ \\
\hline Childhood $\S$ & $1033(63.6)$ & $24(21 ; 30)$ & \\
\hline Adolescence $\ddagger$ & 517 (31.9) & $25(22 ; 30)$ & \\
\hline Adulthood & $73(4.5)$ & $31(26 ; 37.5)$ & \\
\hline No information & $10(0.6)$ & & \\
\hline Total & $1633(100)$ & & \\
\hline
\end{tabular}

Bold indicates statistically significant values. * Kruskal-Wallis test. All other $P$ values were tabulated by using the Mann-Whitney $U$ test. G4/G5 and G2/G3/G4 were not analyzed. Age is statistically different: †Axillary, †̣craniofacial, §palmar, and \|plantar.

Univariable and multivariable analysis of the subjective betterment in the quality of life after surgery are presented in Table 3. In the postoperative period, $95 \%$ of patients reported perceiving betterment in their quality of life. Patients with main craniofacial hyperhidrosis were 10.03 times (adjusted OR, 10.03; 95\% CI, 2.92-34.43) more likely to report a lack of betterment in their quality of life compared with patients with main palmar hyperhidrosis.

The clinical improvement in sweating was great in 1477 patients $(90.4 \%)$, moderate in 107 patients $(6.6 \%)$, and null in 24 patients $(1.5 \%)$. Lost/incomplete information accounted for $1.53 \%(n=25)$ of the medical records.

Table 4 shows the univariable and multivariable analyses of nonimprovement in the main site of sweating after surgery. ORs were calculated for the risk of nonimprovement in sweating at the main hyperhidrosis site (for null clinical improvement). It was not possible to perform a multivariable analysis because of the small number of patients who experienced null clinical improvement (24 patients).

The chance of nonimprovement of sweating at the main site of hyperhidrosis decreased by $12 \%$ with each year of age (OR, $0.88 ; 95 \% \mathrm{CI}, 0.79-0.96)$. The risk of null clinical improvement increased by $27 \%$ with each increase of a BMI unit (OR, 1.27; 95\% CI, 1.09-1.49). 
TABLE 2. Baseline and outcomes data

\begin{tabular}{lc}
\hline \multicolumn{1}{c}{ Variables } & $\mathbf{N}(\%)$ \\
\hline Quality of life before surgery & \\
Very bad & $1313(80.4)$ \\
Bad & $320(19.6)$ \\
Good & $0(0)$ \\
Very good & $0(0)$ \\
Excellent & $0(0)$ \\
Quality of life after surgery & \\
Much worse & $6(0.4)$ \\
Little worse & $14(0.9)$ \\
Equal & $52(3.2)$ \\
Better & $284(17.4)$ \\
Much better & $1277(78.2)$ \\
Clinical improvement & \\
Great & $1477(90.4)$ \\
Moderate & $107(6.6)$ \\
Null & $24(1.5)$ \\
No information & $25(1.5)$ \\
Compensatory hyperhidrosis & \\
Absent & $494(30.3)$ \\
Mild & $698(42.7)$ \\
Moderate & $288(17.6)$ \\
Great & $84(5.1)$ \\
No information & $69(4.2)$ \\
Total & $1633(100)$ \\
\hline
\end{tabular}

The univariable and multivariable analyses of the occurrence and intensity of $\mathrm{CH}$ are presented in Table 5. Severe $\mathrm{CH}$ occurred in 73 patients $(5.1 \%)$, nonsevere $\mathrm{CH}$ occurred in 888 patients $(62.6 \%)$, and it was absent in 459 patients $(32.3 \%)$.

A G2/G3 level resection and greater BMI were most likely to be associated with $\mathrm{CH}$ occurrence. The risk of severe $\mathrm{CH}$ increased by $15 \%$ with each increase of a BMI unit (OR, 1.15; 95\% CI, 1.6-1.25). Age was not associated with $\mathrm{CH}$ or severe $\mathrm{CH}$.

The surgical complications were as follows: 131 patients $(8 \%)$ were diagnosed with pneumothorax and 99 of them ( $6 \%$ of the 1633 patients) needed thoracic drainage for 24 hours to 5 days; 4 patients $(0.25 \%)$ had venous bleeding and 1 patient $(0.06 \%)$ had self-limited arterial bleeding (without the need of reoperation or blood transfusion); $2 \mathrm{pa}-$ tients $(0.12 \%)$ had axillary hematoma; 2 patients $(0.12 \%)$ had bradycardia and 1 patient $(0.06 \%)$ had tachycardia at the immediate postoperative period; 1 patient $(0.06 \%)$ was diagnosed with Horner syndrome (recovered in 6 months); 1 patient $(0.06 \%)$ had thrombophlebitis of thoracic vein; and 7 patients $(0.43 \%)$ had a nonspecified complication.

Our protocol of hospital stay was 24 hours after surgery, but 47 patients $(2.9 \%)$ stayed 2 days, 37 patients $(2.3 \%)$ stayed 3 days, and 5 patients $(0.3 \%)$ stayed 5 days after surgery. All of these patients had extended hospital stay due to voluminous pneumothorax or high-air-fistula.

\section{DISCUSSION}

The potential effect of age on the outcome of sympathectomy as a treatment of hyperhidrosis is a frequent concern in daily surgical practice. Although several factors that affect sympathectomy outcomes have been extensively studied, the effect of age was not clear in the literature. Based on our experience, we hypothesized that sympathectomy outcomes were worse in older patients. However, the present study suggests the opposite: the older the patient is, the greater is the improvement in excessive sweating. Our results support that worse sympathectomy outcomes are more associated with greater BMI and surgical resections that include the G2 level, but not with age.

Our study is one of the biggest studies in primary hyperhidrosis, containing data from 1633 patients. As in other studies, ${ }^{18,19}$ the patients in our study were more likely to be female, in their mid-20s, and with a normal BMI. ${ }^{18,19}$ Patients with main craniofacial hyperhidrosis were always older than patients with other main hyperhidrosis sites. ${ }^{18}$ The sympathectomy level resection is determined by the main hyperhidrosis site; high and multiple levels were avoided, similarly to what was done in other studies. ${ }^{20-22}$

Hyperhidrosis primarily affects young individuals, but onset may occur later in life. Symptoms range from mild and unbothersome to extremely unpleasant. In our study, all patients reported "poor" or "very poor" quality of life before surgery, similar to the what has been observed in most studies that analyzed this topic., ${ }^{2,23}$

Symptom onset in late adulthood or in elderly patients is associated with the possibility of secondary hyperhidrosis. In our study, by design, only patients with primary hyperhidrosis were included; as with other studies, more than one half of our patients had symptom onset during childhood, ${ }^{18,24}$ but a small percentage had adult onset, contributing to a greater age at the time of operation.

Unfortunately, we do not know the exact age of symptoms onset, only the life period. Thus, it was not possible to calculate the exact interval between the age of onset and surgery, and data included only the intensity of symptoms at the first visit to our service. We did not assess whether symptoms worsened or improved during the transition from childhood to adolescence. It is our impression (without specific data to support this hypothesis) that the excessive sweating bothered patients significantly since symptoms onset.

The prevalence of hyperhidrosis decreases as age increases, ${ }^{19,25}$ and this relationship might be the result of good treatment at a younger age, including surgery, adaptation to excessive daily sweating, or amelioration due to an unknown physiological reason. In the epidemiology of hyperhidrosis, age has been extensively 
TABLE 3. Not adjusted and adjusted analysis of nonbetterment in quality of life after surgery

\begin{tabular}{|c|c|c|c|c|c|c|c|c|c|c|}
\hline & \multicolumn{2}{|c|}{ Quality of life after surgery } & \multirow{3}{*}{$\begin{array}{c}\text { OR not } \\
\text { adjusted }\end{array}$} & \multirow{2}{*}{\multicolumn{2}{|c|}{ CI $(95 \%)$}} & \multirow[b]{3}{*}{$P$ value } & \multirow{3}{*}{$\begin{array}{c}\text { OR } \\
\text { adjusted }\end{array}$} & \multirow{2}{*}{\multicolumn{2}{|c|}{ CI $(95 \%)$}} & \multirow[b]{3}{*}{$P$ value } \\
\hline & \multirow{2}{*}{$\begin{array}{c}\text { Better } \\
\text { n }(\%)\end{array}$} & \multirow{2}{*}{$\begin{array}{c}\text { Not better } \\
\mathrm{n}(\%)\end{array}$} & & & & & & & & \\
\hline & & & & Lower & Upper & & & Lower & Upper & \\
\hline \multicolumn{11}{|l|}{ Sex } \\
\hline Female & $1054(96.3)$ & $41(3.7)$ & 1.00 & & & & & & & \\
\hline Male & $507(94.2)$ & $31(5.8)$ & 1.57 & 0.97 & 2.54 & .064 & & & & \\
\hline \multicolumn{11}{|l|}{ Main hyperhidrosis site } \\
\hline Palmar & $984(97.1)$ & $29(2.9)$ & 1.00 & & & & 1.00 & & & \\
\hline Axillary & $531(93.3)$ & $38(6.7)$ & 2.43 & 1.48 & 3.98 & $<.001$ & 1.79 & 0.94 & 3.41 & .075 \\
\hline Craniofacial & $26(83.9)$ & $5(16.1)$ & 6.53 & 2.34 & 18.20 & $<.001$ & 10.03 & 2.92 & 34.43 & $<.001$ \\
\hline Plantar & $20(100.0)$ & $0(0.0)$ & $*$ & & & .998 & * & & & .998 \\
\hline \multicolumn{11}{|l|}{ Technique } \\
\hline $\mathrm{G} 2$ & $67(97.1)$ & $2(2.9)$ & 0.61 & 0.14 & 2.61 & .502 & 0.29 & 0.05 & 1.76 & .180 \\
\hline G3 & 519 (97.6) & $13(2.4)$ & 0.51 & 0.26 & 1.00 & .049 & 0.71 & 0.32 & 1.58 & .403 \\
\hline G4 & $549(95.3)$ & $27(4.7)$ & 1.00 & & & & 1.00 & & & \\
\hline $\mathrm{G} 2 / \mathrm{G} 3$ & $246(95.3)$ & $12(4.7)$ & 0.99 & 0.49 & 1.99 & .982 & 1.09 & 0.46 & 2.58 & .842 \\
\hline G3/G4 & $168(91.3)$ & $16(8.7)$ & 1.94 & 1.02 & 3.68 & .044 & 1.89 & 1.00 & 3.61 & .052 \\
\hline \multicolumn{11}{|l|}{ Age of symptom onset } \\
\hline Childhood & $1000(96.8)$ & $33(3.2)$ & 1.00 & & & & & & & \\
\hline Adolescence & $485(93.8)$ & $32(6.2)$ & 2.00 & 1.22 & 3.29 & .006 & & & & \\
\hline Adulthood & $66(90.4)$ & $7(9.6)$ & 3.21 & 1.37 & 7.54 & .007 & & & & \\
\hline Age, y, median (p25; p75) & $25(21 ; 30)$ & $25(22 ; 31)$ & 1.00 & 0.97 & 1.04 & .794 & & & & \\
\hline $\begin{array}{l}\text { BMI, } \mathrm{kg} / \mathrm{m}^{2}, \text { median } \\
\quad(\mathrm{p} 25 ; \mathrm{p} 75)\end{array}$ & $21.7(20.1 ; 23.6)$ & $21.9(20 ; 24.5)$ & 1.06 & 0.97 & 1.17 & .223 & & & & \\
\hline
\end{tabular}

Bivariable logistic regression and multivariable logistic regression with backward stepwise selection criteria. Bold indicates statistically significant values. G4/G5 and G2/G3/G4 were not analyzed. $O R$, Odds ratio; $C I$, confidence interval; BMI, body mass index. *OR could not be calculated.

studied (eg, symptom topography vs age of symptoms onset), ${ }^{26-28}$ and the reason why fewer older individuals seek treatment has also been explored.

For all patients seeking medical (ie, noninvasive) treatment for hyperhidrosis, a very popular initial choice is the oral use of oxybutynin. ${ }^{27}$ This treatment is frequently selected for older patients who frequently have other comorbidities that increase surgical risk. ${ }^{27}$ Many of these patients already use other daily medications to control their concomitant diseases, and the addition of an oxybutynin tablet to this routine does not generate some of the discomfort reported by younger patients, who complain about the daily use of the medication. The oldest patient in our sample was 70 years old, indicating that the symptoms might be irritating enough to motivate even elderly patients to accept the risk of surgery. We can speculate that older patients might not be offered treatment sooner due to the lack of referral in the health care system.

It is likely that surgery risk represents a weak factor in the decision to undergo the operation and that the expectation of achieving outcomes as good as those observed in younger patients is more important during the decision-making process. Patient age at surgery and variations in the quality of life and/or improvement in sweating, however, have been understudied.
In the postoperative period, we observed a greater perception of betterment in the quality of life. Quality of life in patients with hyperhidrosis is an important concern when it comes to the study of treatment options for this condition. ${ }^{14,29,30}$ Wolosker and colleagues ${ }^{27}$ reported betterment of $77 \%$ in the quality of life of patients older than 40 years after clinical treatment with oxybutynin, which was even greater in patients older than 50 years $(87.1 \%)$. In agreement with our results, other authors reported great betterment in the quality of life after sympathectomy. ${ }^{5,14,29,31}$ The only significant risk factor of not experiencing betterment of the quality of life was the craniofacial region as the main hyperhidrosis site. Other authors have not reported this finding. Upper sympathectomy resection levels for the treatment of craniofacial hyperhidrosis may explain that result.

In agreement with previous studies, ${ }^{26-28}$ our data showed that the craniofacial region as the main site of hyperhidrosis is associated with older patients, but age was not found to be a significant factor that may block the quality of life betterment. We emphasize that although it was statistically significant, the number of patients with craniofacial hyperhidrosis was proportionally lower in this sample. 
TABLE 4. Not adjusted and adjusted analysis of clinical improvement

\begin{tabular}{|c|c|c|c|c|c|c|c|c|c|c|}
\hline & \multicolumn{2}{|c|}{ Clinical improvement } & \multirow{3}{*}{$\begin{array}{c}\text { OR not } \\
\text { adjusted }\end{array}$} & \multirow{2}{*}{\multicolumn{2}{|c|}{ CI $(95 \%)$}} & \multirow[b]{3}{*}{$P$ value } & \multirow{3}{*}{$\begin{array}{c}\text { OR } \\
\text { adjusted }\end{array}$} & \multirow{2}{*}{\multicolumn{2}{|c|}{ CI $(95 \%)$}} & \multirow[b]{3}{*}{$P$ value } \\
\hline & \multirow{2}{*}{$\frac{\text { Moderate/great }}{\text { n }(\%)}$} & \multirow{2}{*}{$\begin{array}{c}\text { Null } \\
\text { n }(\%)\end{array}$} & & & & & & & & \\
\hline & & & & Lower & Upper & & & Lower & Upper & \\
\hline \multicolumn{11}{|l|}{ Sex } \\
\hline Female & $1078(98.4)$ & $17(1.6)$ & 1.00 & & & & & & & \\
\hline Male & $531(98.7)$ & $7(1.3)$ & 0.84 & 0.35 & 2.03 & .692 & & & & \\
\hline \multicolumn{11}{|l|}{ Main hyperhidrosis site } \\
\hline Palmar & 999 (98.6) & $14(1.4)$ & 1.00 & & & & & & & \\
\hline Axillary & $559(98.2)$ & $10(1.8)$ & 1.28 & 0.56 & 2.89 & .559 & & & & \\
\hline Craniofacial & $31(100.0)$ & $0(0.0)$ & $*$ & & & .998 & & & & \\
\hline Plantar & $20(100.0)$ & $0(0.0)$ & $*$ & & & .998 & & & & \\
\hline \multicolumn{11}{|l|}{ Technique } \\
\hline $\mathrm{G} 2$ & 68 (98.6) & $1(1.4)$ & 0.69 & 0.09 & 5.40 & .725 & & & & \\
\hline G3 & $527(99.1)$ & $5(0.9)$ & 0.45 & 0.16 & 1.27 & .132 & & & & \\
\hline G4 & $564(97.9)$ & $12(2.1)$ & 1.0 & & & & & & & \\
\hline $\mathrm{G} 2 / \mathrm{G} 3$ & $255(98.8)$ & $3(1.2)$ & 0.55 & 0.16 & 1.98 & .362 & & & & \\
\hline G3/G4 & $182(98.9)$ & $2(1.1)$ & 0.52 & 0.12 & 2.33 & .390 & & & & \\
\hline \multicolumn{11}{|l|}{ Age of symptom onset } \\
\hline Childhood & $1020(98.7)$ & $13(1.3)$ & & & & & & & & \\
\hline Adolescence & $506(97.9)$ & $11(2.1)$ & 1.71 & 0.76 & 3.83 & .196 & & & & \\
\hline Adulthood & $73(100.0)$ & $0(0.0)$ & 0.00 & 0.00 & $\dagger$ & .997 & & & & \\
\hline Age, $y$, median $(\mathrm{p} 25 ; \mathrm{p} 75)$ & $25(21 ; 30)$ & $23(21.3 ; 25.5)$ & 0.91 & 0.84 & 0.99 & .022 & 0.88 & 0.79 & 0.96 & .007 \\
\hline $\begin{array}{l}\text { BMI, } \mathrm{kg} / \mathrm{m}^{2}, \text { median } \\
\quad(\mathrm{p} 25 ; \mathrm{p} 75)\end{array}$ & $21.7(20.1 ; 23.6)$ & $22.7(20.3 ; 24.6)$ & 1.18 & 1.01 & 1.37 & .033 & 1.27 & 1.09 & 1.49 & .003 \\
\hline
\end{tabular}

Bivariable logistic regression and multivariable logistic regression with backward stepwise selection criteria. Bold indicates statistically significant values. G4/G5 and G2/G3/G4 were not analyzed. $O R$, Odds ratio; $C I$, confidence interval; BMI, body mass index. *OR could not be calculated. $\dagger C I$ could not be calculated.

In this study, 24 patients $(1.46 \%)$ reported no improvement in sweating after sympathectomy, which is within the expected results according to the literature, ie, the incidence of technical failure was less than $8 \%{ }^{6,23}$ In addition, we observed that older and thinner patients experienced a greater perception of improvement in sweating in the main site of hyperhidrosis. Therefore, surgical treatment later in life does not affect the benefits of surgery and leads to a greater clinical improvement. Greater BMI is a well-known factor that increases technical difficulties due to the similarities between fat and the sympathetic ganglion.

$\mathrm{CH}$ potentially worsens postoperative quality of life of patients who undergo sympathectomy for the treatment of primary hyperhidrosis, and many authors consider it the worst long-term side effect of sympathectomy. $\mathrm{CH}$ is difficult to treat ${ }^{32}$; thus, when $\mathrm{CH}$ occurs less frequently, patients tend to be more satisfied. Only resections in G2/G3 level and greater BMI represented a greater risk of $\mathrm{CH}$ in our study. Sympathectomy ganglion resection at greater levels and resection at more than one ganglionic level in the same surgery have a well-known association with a great incidence of severe $\mathrm{CH}$ and no difference in symptom resolution, and incidence of dry hands and gustatory sweating. ${ }^{7,22,33}$ Therefore, we avoided performing multiple resections in our patient population and preferred sympathectomy at the G4 or G3 level, as demonstrated in this study with $35.5 \% \mathrm{G} 4$ and $32.8 \% \mathrm{G} 3$ surgeries. The resection level is determined by the main site of hyperhidrosis. In elderly patients, we found a greater incidence of resections at the G2 and G2-G3 levels, accompanying the greater prevalence of craniofacial hyperhidrosis as the main site in this population. Fukuda and colleagues $^{28}$ verified that $100 \%$ of patients reported some degree of craniofacial sweating improvement when submitted to G2, almost $95 \%$ when submitted to G3 and nearly $80 \%$ when submitted to G4 sympathectomy for craniofacial hyperhidrosis, at the expense of a high frequency of $\mathrm{CH}$ with greater levels of sympathectomy.

Multiple and higher levels of sympathectomy denervation are a well-known risk factor for $\mathrm{CH}^{20-22}$ as well as BMI. ${ }^{34}$ Previous studies support the direct influence of BMI on surgery outcome and the increased occurrence of $\mathrm{CH}$ and severe $\mathrm{CH}$ in patients who are obese in the postoperative period. ${ }^{34,35}$

Gallagher and colleagues ${ }^{36}$ demonstrated that the percentage of body fat increases with age in both women and men due to a change in the fat accumulation process that occurs over the years, which may explain why older patients tend to have greater BMI values. Our team prioritizes surgeries in patients with normal BMI or few individuals with increased BMI due to increased muscle mass but not fat accumulation. ${ }^{22,37}$ As there were few patients with 
TABLE 5. Not adjusted and adjusted analysis of presence and intensity of compensatory hyperhidrosis

\begin{tabular}{|c|c|c|c|c|c|c|c|c|c|c|}
\hline & \multirow{2}{*}{$\frac{\text { Absent }}{\text { n (\%) }}$} & \multirow{2}{*}{$\frac{\text { Present }}{\text { n }(\%)}$} & \multirow{2}{*}{$\begin{array}{c}\text { OR not } \\
\text { adjusted }\end{array}$} & \multicolumn{2}{|c|}{ CI $(95 \%)$} & \multirow[b]{2}{*}{$P$ value } & \multirow{2}{*}{$\begin{array}{c}\text { OR } \\
\text { adjusted }\end{array}$} & \multicolumn{2}{|c|}{ CI $(95 \%)$} & \multirow[b]{2}{*}{$P$ value } \\
\hline & & & & Lower & Upper & & & Lower & Upper & \\
\hline \multicolumn{11}{|l|}{ Compensatory hyperhidrosis } \\
\hline \multicolumn{11}{|l|}{ Sex } \\
\hline Female & $357(33.6)$ & $704(66.4)$ & 1.00 & & & & & & & \\
\hline Male & 137 (26.9) & $372(73.1)$ & 1.38 & 1.09 & 1.74 & .007 & & & & \\
\hline \multicolumn{11}{|l|}{ Main hyperhidrosis site } \\
\hline Palmar & $298(30.7)$ & $672(69.3)$ & 1.00 & & & & & & & \\
\hline Axillary & 185 (33.6) & $365(66.4)$ & 0.88 & 0.70 & 1.09 & .241 & & & & \\
\hline Craniofacial & $6(20.0)$ & $24(80.0)$ & 1.77 & 0.72 & 4.38 & .214 & & & & \\
\hline Plantar & $5(25.0)$ & $15(75.0)$ & 1.33 & 0.48 & 3.69 & .584 & & & & \\
\hline \multicolumn{11}{|l|}{ Technique } \\
\hline G2 & $13(21.3)$ & $48(78.7)$ & 2.00 & 1.06 & 3.79 & .033 & 1.35 & 0.68 & 2.68 & .399 \\
\hline G3 & $166(31.9)$ & $355(68.1)$ & 1.16 & 0.90 & 1.49 & .25 & 1.10 & 0.85 & 1.43 & .457 \\
\hline G4 & $198(35.2)$ & $365(64.8)$ & 1.00 & & & & 1.00 & & & \\
\hline G2/G3 & $60(25.3)$ & 177 (74.7) & 1.60 & 1.14 & 2.25 & .007 & 1.80 & 1.21 & 2.69 & .004 \\
\hline G3/G4 & $55(31.1)$ & $122(68.9)$ & 1.20 & 0.84 & 1.73 & .317 & 1.05 & 0.71 & 1.55 & .817 \\
\hline \multicolumn{11}{|l|}{ Age of symptom onset } \\
\hline Childhood & $303(30.5)$ & $689(69.5)$ & 1.00 & & & & & & & \\
\hline Adolescence & $154(30.7)$ & $347(69.3)$ & 0.99 & 0.79 & 1.25 & .939 & & & & \\
\hline Adulthood & $30(44.1)$ & $38(55.9)$ & 0.56 & 0.34 & 0.92 & .021 & & & & \\
\hline Age, $y$, median $(\mathrm{p} 25 ; \mathrm{p} 75)$ & $25(21 ; 30)$ & $25(21 ; 30)$ & 1.01 & 1.00 & 1.02 & .346 & & & & \\
\hline $\begin{array}{l}\text { BMI, } \mathrm{kg} / \mathrm{m}^{2}, \text { median } \\
\quad(\mathrm{p} 25 ; \mathrm{p} 75)\end{array}$ & $21.5(19.9 ; 23.5)$ & $21.8(20.1 ; 23.7)$ & 1.05 & 1.00 & 1.09 & .039 & 1.05 & 1.00 & 1.09 & .045 \\
\hline Severe $\mathbf{C H}$ & Nonsevere & Severe & & & & & & & & \\
\hline \multicolumn{11}{|l|}{ Sex } \\
\hline Female & $651(93.5)$ & $45(6.5)$ & 1.00 & & & & & & & \\
\hline Male & $332(89.5)$ & $39(10.5)$ & 1.70 & 1.09 & 2.66 & .021 & & & & \\
\hline \multicolumn{11}{|l|}{ Main hyperhidrosis site } \\
\hline Palmar & $622(93.5)$ & $43(6.5)$ & 1.00 & & & & & & & \\
\hline Axillary & $327(90.1)$ & $36(9.9)$ & 1.59 & 1.00 & 2.53 & .049 & & & & \\
\hline Craniofacial & $20(83.3)$ & $4(16.7)$ & 2.89 & 0.95 & 8.84 & .062 & & & & \\
\hline Plantar & $14(93.3)$ & $1(6.7)$ & 1.03 & 0.13 & 8.04 & .975 & & & & \\
\hline \multicolumn{11}{|l|}{ Technique } \\
\hline G2 & $45(93.8)$ & $3(6.2)$ & 0.92 & 0.27 & 3.10 & .889 & & & & \\
\hline G3 & $325(92.1)$ & $28(7.9)$ & 1.01 & 0.59 & 1.71 & .979 & & & & \\
\hline G4 & 333 (91.7) & $30(8.3)$ & 1.00 & & & & & & & \\
\hline G2/G3 & $163(93.7)$ & $11(6.3)$ & 0.88 & 0.43 & 1.78 & .711 & & & & \\
\hline G3/G4 & $110(91.7)$ & $10(8.3)$ & 1.08 & 0.51 & 2.25 & .848 & & & & \\
\hline \multicolumn{11}{|l|}{ Age of symptom onset } \\
\hline Childhood & 937 (94.8) & $51(5.2)$ & 1.00 & & & & & & & \\
\hline Adolescence & $471(94.4)$ & $28(5.6)$ & 1.09 & 0.68 & 1.76 & .715 & & & & \\
\hline Adulthood & $64(94.1)$ & $4(5.9)$ & 1.15 & 0.40 & 3.28 & .796 & & & & \\
\hline Age, $y$, median (p25; 775$)$ & $25(21 ; 30)$ & $25.5(22 ; 33.8)$ & 1.03 & 1.00 & 1.06 & .023 & & & & \\
\hline $\begin{array}{l}\text { BMI, } \mathrm{kg} / \mathrm{m}^{2}, \text { median } \\
\quad(\mathrm{p} 25 ; \mathrm{p} 75)\end{array}$ & $21.6(20.1 ; 23.5)$ & $22.8(20.6 ; 25)$ & 1.14 & 1.05 & 1.24 & .002 & 1.15 & 1.06 & 1.25 & .001 \\
\hline
\end{tabular}

$\overline{\text { Bivariable logistic regression and Multivariable logistic regression with backward stepwise selection criteria. G4/G5 and G2/G3/G4 were not analyzed. Bold indicates statistically }}$ significant values. $O R$, Odds ratio; $C I$, confidence interval; $B M I$, body mass index; $\mathrm{CH}$, compensatory hyperhidrosis.

BMI greater than $28 \mathrm{~kg} / \mathrm{m}^{2}$, we could not draw any conclusions about the impact of overweight in $\mathrm{CH}$.

The prevalence of severe $\mathrm{CH}(5.4 \%)$ in our patient population is consistent with previous studies. ${ }^{11,38}$ The median age of patients with $\mathrm{CH}$ and severe $\mathrm{CH}$ were similar in our study, and age had no association with the occurrence of $\mathrm{CH}$ or severe $\mathrm{CH}$.

The present study has some limitations. As a retrospective study based on a review of medical records, a small amount of information was lost due to incomplete data 
obtained during medical visits, despite our well-structured study protocol that was consistently followed for many years. In addition, clinical improvement in the primary sweating site was evaluated according to the patient's judgment, not by an objective evaluation with a sudorometer (such as a VapoMeter). ${ }^{39}$ Another limitation is that only 24 patients reported no improvement in excessive sweating, and therefore some associations may have been missed. Furthermore, as the postoperative questionnaire evaluated perceived betterment and was not a direct comparison with the preoperative questionnaire, some statistical power may have been lost.

Despite these limitations, we believe this study with data from a large patient population provides valuable information that may help older patients decide whether they should undergo sympathectomy. The results of our study showed that age was associated with improvement in clinical sweating at the main hyperhidrosis site, and that $\mathrm{CH}$ and a lack of reported quality of life betterment were related to other variables (main site of hyperhidrosis, BMI, and level of resection in sympathectomy). According to our results, older patients with noncraniofacial hyperhidrosis and a BMI closer to normal tend to have excellent outcomes with surgery.

\section{CONCLUSIONS}

We concluded that patients at an old age reported an improvement in clinical sweating in the main affected site of hyperhidrosis after sympathectomy. Other sympathectomy outcomes (ie, quality of life and $\mathrm{CH}$ occurrence) appear to be similar between older and younger patients.

\section{Conflict of Interest Statement}

Authors have nothing to disclose with regard to commercial support.

\section{References}

1. Cameron AEP. Selecting the right patient for surgical treatment of hyperhidrosis. Thorac Surg Clin. 2016;26:403-6.

2. Wolosker N, de Campos JRM, Kauffman P, de Oliveira LA, Munia MAS, Jatene FB. Evaluation of quality of life over time among 453 patients with hyperhidrosis submitted to endoscopic thoracic sympathectomy. J Vasc Surg. 2012;55: 154-6.

3. Neves S, Uchoa PC, Wolosker N, Munia MA, Kauffman P, de Campos JRM, et al. Long-term comparison of video-assisted thoracic sympathectomy and clinical observation for the treatment of palmar hyperhidrosis in children younger than 14. Pediatr Dermatol. 2012;29:575-9.

4. Buraschi J. Videothoracoscopic sympathicolysis procedure for primary palmar hyperhidrosis in children and adolescents [in Spanish]. Arch Argent Pediatr. 2008; 106:32-5.

5. Dias LI, Miranda ECM, Toro IFC, Mussi RK. Relationship between anxiety, depression and quality of life with the intensity of reflex sweating after thoracoscopic sympathectomy for treatment of primary hyperhidrosis [in Portuguese]. Rev Col Bras Cir. 2016;43:354-9.

6. Dharmaraj B, Kosai NR, Gendeh H, Ramzisham AR, Das S. A prospective cohort study on quality of life after endoscopic thoracic sympathectomy for primary hyperhidrosis. Clin Ter. 2016;167:67-71.

7. Ong W, Lee A, Tan WB, Lomanto D. Long-term results of a randomized controlled trial of T2 versus T2-T3 ablation in endoscopic thoracic sympathectomy for palmar hyperhidrosis. Surg Endosc. 2016;30:1219-25.
8. Teivelis MP, Varella AY, Wolosker N. Expanded level of sympathectomy and incidence or severity of compensatory hyperhidrosis. J Thorac Cardiovasc Surg. 2014;148:2443-4.

9. Lee SS, Lee YU, Lee J-H, Lee JC. Comparison of the long-term results of R3 and R4 sympathicotomy for palmar hyperhidrosis. Korean J Thorac Cardiovasc Surg. 2017;50:197-201

10. Wolosker N, de Campos JRM, Kauffman P, Puech-Leão P. A randomized placebo-controlled trial of oxybutynin for the initial treatment of palmar and axillary hyperhidrosis. J Vasc Surg. 2012;55:1696-700.

11. Teivelis MP, Wolosker N, Krutman M, Milanez de Campos JR, Kauffman P, Puech-Leão P. Compensatory hyperhidrosis: results of pharmacologic treatment with oxybutynin. Ann Thorac Surg. 2014;98:1797-802.

12. de Andrade Filho LO, Kuzniec S, Wolosker N, Yazbek G, Kauffman P, Milanez de Campos JR. Technical difficulties and complications of sympathectomy in the treatment of hyperhidrosis: an analysis of 1731 cases. Ann Vasc Surg. 2013;27: 447-53.

13. de Campos JRM, Kauffman P, Werebe EC. Questionnaire of quality of life in patients with primary hyperhidrosis. J Bras Pneumol. 2003;1:171-81.

14. de Campos JRM, Kauffman P, Werebe Ede C, Andrade Filho LO, Kusniek S, Wolosker N, et al. Quality of life, before and after thoracic sympathectomy: report on 378 operated patients. Ann Thorac Surg. 2003;76:886-91.

15. Varella AYM, Fukuda JM, Teivelis MP, Campos JR, Kauffman P, Cucato GG, et al. Translation and validation of Hyperhidrosis Disease Severity Scale. Rev Assoc Med Bras (1992). 2016;62:843-7.

16. Amir M, Arish A, Weinstein Y, Pfeffer M, Levy Y. Impairment in quality of life among patients seeking surgery for hyperhidrosis (excessive sweating): preliminary results. Isr J Psychiatry Relat Sci. 2000;37:25-31.

17. Yazbek G, Wolosker N, de Campos JRM, Kauffman P, Ishy A, Puech-Leão P Palmar hyperhidrosis - which is the best level of denervation using videoassisted thoracoscopic sympathectomy: T2 or T3 ganglion? J Vasc Surg. 2005; 42:281-5.

18. Estevan FA, Wolosker MB, Wolosker N, Puech-Leão P. Epidemiologic analysis of prevalence of the hyperhidrosis. An Bras Dermatol. 2017;92:630-4.

19. Lear W, Kessler E, Solish N, Glaser DA. An epidemiological study of hyperhidrosis. Dermatol Surg. 2007;33:S69-75.

20. Zhang W, Wei Y, Jiang H, Xu J, Yu D. R3 versus R4 thoracoscopic sympathectomy for severe palmar hyperhidrosis. Thorac Cardiovasc Surg. 2017;65:491-6.

21. Munia MAS, Wolosker N, Kaufmann P, de Campos JRM, Puech-Leão P. Sustained benefit lasting one year from T4 instead of T3-T4 sympathectomy for isolated axillary hyperhidrosis. Clinics (Sao Paulo). 2008;63:771-4.

22. Zhang W, Yu D, Wei Y, Xu J, Zhang X. A systematic review and meta-analysis of T2, T3 or T4, to evaluate the best denervation level for palmar hyperhidrosis. Sci Rep. 2017;7:129.

23. Munia MAS, Wolosker N, Kauffman P, de Campos JR, Puech-Leão P. A randomized trial of T3-T4 versus T4 sympathectomy for isolated axillary hyperhidrosis $J$ Vasc Surg. 2007;45:130-3.

24. Tu Y-R, Li X, Lin M, Lai F-C, Li Y-P, Chen J-F, et al. Epidemiological survey of primary palmar hyperhidrosis in adolescent in Fuzhou of People's Republic of China. Eur J Cardiothorac Surg. 2007;31:737-9.

25. Haider A, Solish N. Focal hyperhidrosis: diagnosis and management. CMAJ. 2005; 172:69-75.

26. Wolosker N, Teivelis MP, Krutman M, Campbell TPD de A, Kauffman P, de Campos JR, et al. Long-term results of oxybutynin use in treating facial hyperhidrosis. An Bras Dermatol. 2014;89:912-6.

27. Wolosker N, Krutman M, Teivelis MP, Paula RP, Kauffman P, de Campos JRM, et al. Analysis of oxybutynin treatment for hyperhidrosis in patients aged over 40 years. Einstein (Sao Paulo). 2014;12:42-7.

28. Fukuda JM, Varella AYM, Teivelis MP, de Campos JRM, Kauffman P, Pinheiro LL, et al. Video-assisted thoracoscopic sympathectomy for facial hyperhidrosis: the influence of the main site of complaint. Ann Vasc Surg. 2017;46: 337-44.

29. Wolosker N, Yazbek G, de Campos JRM, Munia MA, Kauffman P, Jatene FBB, et al. Quality of life before surgery is a predictive factor for satisfaction among patients undergoing sympathectomy to treat hyperhidrosis. J Vasc Surg. 2010; 51:1190-4.

30. Campanati A, Penna L, Guzzo T, Menotta L, Silvestri B, Lagalla G, et al. Quality-of-life assessment in patients with hyperhidrosis before and after treatmen with botulinum toxin: results of an open-label study. Clin Ther. 2003;25:298-308.

31. Lembrança L, Wolosker N, de Campos JRM, Kauffman P, Teivelis MP, PuechLeão P. Videothoracoscopic sympathectomy results after oxybutynin chloride treatment failure. Ann Vasc Surg. 2017;43:283-7. 
32. Weksler B. Is there light at the end of the tunnel for patients with severe compensatory hyperhidrosis? J Thorac Cardiovasc Surg. 2017;154:e117.

33. Ribas Milanez de Campos J, Kauffman P, Wolosker N, Munia MA, de Campos Werebe E, Andrade Filho LO, et al. Axillary hyperhidrosis: T3/T4 versus T4 thoracic sympathectomy in a series of 276 cases. J Laparoendosc Adv Surg Tech A. 2006;16:598-603.

34. de Campos JRM, Wolosker N, Takeda FR, Kauffman P, Kuzniec S, Jatene FB, et al. The body mass index and level of resection: predictive factors for compensatory sweating after sympathectomy. Clin Auton Res. 2005;15:116-20.

35. Dobosz L, Cwalina N, Stefaniak T. Influence of body mass index on compensatory sweating in patients after thoracic sympathectomy due to palmar hyperhidrosis. Thorac Cardiovasc Surg. 2017;65:497-502.

36. Gallagher D, Visser M, Sepúlveda D, Pierson RN, Harris T, Heymsfield SB. How useful is body mass index for comparison of body fatness across age, sex, and ethnic groups? Am J Epidemiol. 1996;143: 228-39.

37. Zhang W, Wei Y, Jiang H, Xu J, Yu D. T3 versus T4 thoracoscopic sympathicotomy for palmar hyperhidrosis: a meta-analysis and system review. J Surg Res. 2017;218:124-31.

38. Vannucci F, Araújo JA. Thoracic sympathectomy for hyperhidrosis: from surgical indications to clinical results. J Thorac Dis. 2017;9:S178-92.

39. Wolosker N, Ishy A, Yazbek G, de Campos JRM, Kauffman P, Puech-Leão P, et al. Objective evaluation of plantar hyperhidrosis after sympathectomy. Clinics (Sao Paulo). 2013;68:311-5.

Key Words: thoracoscopy, sweating, compensatory sweating, hyperhidrosis, sympathectomy, age 